\title{
The Influence of Different Testing Time for Stone Radioactive Detection Results
}

\author{
Xiang Yu \\ Chongqing Academy of Metrology and Quality Inspection, Chongqing, 40112, China
}

Email:20227497@qq.com

Key words: Radioactivity; Detection time; Uncertainty

\begin{abstract}
In order to understand the different detection time of stone radioactive detection results impact, this paper for the three groups of stone sample take the different detection time radioactivity survey, and the detection results are analyzed. The best time of radioactive detection of the proposed stone is proposed. And on the premise of fully ensure the accuracy of the detection, provide the basis for maximizing detection efficiency.
\end{abstract}

\section{Introduction}

GB 6566-2010 Radical Nuclide Limit for Building Materials ${ }^{[1]}$ is a mandatory standard for control of radical nuclides in building materials in China. The standard applies to inorganic non-metallic building materials used in various buildings, including building materials mixed with industrial waste residues. In addition to detection time of different building materials (radioactive level is not the same), detection equipment, shape and number of samples and testing methods are clearly defined in the standard. Radioactive ray strength is not constant, so the detection time should be determined based on the experience of detection personnel and different types of building materials. In general, the longer the test time is, the more ideal the test result will be. However, the work mode is not very scientific and the detection time is too long to improve the detection efficiency.

Stone products are processed by their own natural ore, so its radioactivity mainly depends on radioactive level of the raw ore, and the ore origin, collection location and other factors will affect the radioactive level of the processed stone. Because product test results are scattered, only scientific development of detection time can effectively guarantee the test data. Therefore, a large number of radioactive tests at different detection time have been carried out for stone samples representing different radioactivity levels in three categories in the Thesis. Impact of detection time of different stones and different radioactive level on the test results can be can understood through analysis of the data and uncertainty of the test results, so as to scientifically and rationally develop the detection time to guide the actual detection operate accurately and efficiently.

\section{Test method}

\subsection{Instruments}

Low background multi-channel $r$ spectrometer, electronic balance, drying oven

2.2 Test methods

First, the instrument was preheated for more than $60 \mathrm{~min}$. Background, radium-226 standard source, thorium-232 standard source and potassium-40 radioactive source were continuously sampled to complete calibration of equipment parameters according to the equipment requirements, and radioactivity of dried stone samples was measured to obtain specific radioactivity of radium-226, thorium-232 and potassium-40. The measurement results were counted and uncertainty of measurement was analyzed. 


\section{Mathematical model}

The classical mathematical model used to measure internal and external illumination index is established according to stipulation of the test method and test procedure, and the relative standard uncertainty evaluation method is adopted. Modeling is carried out as follows, including model of internal and external exposure index:

$$
I_{R a}=\frac{C_{R a}}{200}
$$

$\mathrm{I}_{\mathrm{Ra}}$ refers to internal exposure index; $\mathrm{C}_{\mathrm{Ra}}$ refers to radioactivity specific activity of the natural radionuclide $\mathrm{Ra}$ in stone in $\mathrm{Bq} / \mathrm{kg} ; 200$ refers to the specific activity limit of radionuclide $\mathrm{Ra}$ specified in GB/T6566-2010 Radical Nuclide Limit for Building Materials, in Bq/kg.

$$
I_{r}=\frac{C_{R a}}{370}+\frac{C_{T h}}{260}+\frac{C_{K}}{420}
$$

Ir refers to external exposure index; $\mathrm{C}_{\mathrm{Ra}}, \mathrm{C}_{\mathrm{Th}}$ and $\mathrm{C}_{\mathrm{K}}$ refer to radioactivity specific activities of natural radionuclide 226Ra, 232Th and 40K in the stone, in the unit of Bq/kg; 370, 260 and 4200 refer to the specific activity limit specified in GB/T6566-2010 Radical Nuclide Limit for Building Materials only in consideration of external exposure, in $\mathrm{Bq} / \mathrm{kg}$.

\section{Test and data analysis}

\subsection{Experimental data}

4.1.1 The radioactive standard sample was measured for the No. \#1 stone product, and the measurement times are the common detection time of 600s, 1600s and 3600s respectively; the number of measurements is 20 times. Data statistical results of measurement for 20 times are shown in Table 1.

Table .1 Radioactive measurement data statistics results of $1 \#$ stone samples

\begin{tabular}{ccccccc}
\hline \multirow{2}{*}{ Measurement time/s } & \multicolumn{3}{c}{ Internal exposure index } & \multicolumn{3}{c}{ External exposure index } \\
\cline { 2 - 7 } & Average value & Range & Mean of range ratio/\% & Average value & Range & Mean of range ratio/\% \\
\hline 600 & 0.124 & 0.11 & 85 & 0.508 & 0.25 & 49 \\
1800 & 0.114 & 0.06 & 49 & 0.503 & 0.14 & 27 \\
3600 & 0.119 & 0.04 & 35 & 0.512 & 0.14 & 28 \\
\hline
\end{tabular}

4.1.2 The radioactive standard sample was measured for the No. \#2 stone product, and the measurement times are the common detection time of $600 \mathrm{~s}, 1600 \mathrm{~s}$ and $3600 \mathrm{~s}$ respectively; the number of measurements is 20 times. Data statistical results of measurement for 20 times are shown in Table 2.

Table .2 Radioactive measurement data statistics results of 2\# stone samples

\begin{tabular}{ccccccc}
\hline \multirow{2}{*}{ Measurement time/s } & \multicolumn{3}{c}{ Internal exposure index } & \multicolumn{3}{c}{ External exposure index } \\
\cline { 2 - 7 } & Average value & Range & & Average value & Range \\
\hline 600 & 0.530 & 0.13 & 25 & 1.014 & 0.22 & 22 \\
1800 & 0.501 & 0.11 & 22 & 1.031 & 0.23 & 22 \\
3600 & 0.499 & 0.15 & 31 & 1.009 & 0.28 & 28 \\
\hline
\end{tabular}

4.1.3 The radioactive standard sample was measured for the No. \#3 stone product, and the measurement times are the common detection time of 600s, 1600s and 3600s respectively; the number of measurements is 20 times. Data statistical results of measurement for 20 times are shown in Table 2. 
Table .3 Radioactive measurement data statistics results of $3 \#$ stone samples

\begin{tabular}{ccccccc}
\hline \multirow{2}{*}{ Measurement time/s } & \multicolumn{3}{c}{ Internal exposure index } & \multicolumn{3}{c}{ External exposure index } \\
\cline { 2 - 7 } & Average value & Range & & Average value & Range \\
\hline 600 & 1.171 & 0.19 & 16 & 2.149 & 0.47 & 22 \\
1800 & 1.162 & 0.16 & 14 & 2.146 & 0.35 & 16 \\
3600 & 1.176 & 0.13 & 11 & 2.158 & 0.30 & 14 \\
\hline
\end{tabular}

\subsection{Analysis for uncertainty of measurement}

\subsubsection{Sources for uncertainty of measurement}

In comprehensive consideration of factors such as equipment, personnel, environment and measurement methods, the sources of uncertainty of measurement include that a variety of random factors to make the data collection results not repeated on measurement, uncertainty in the measurement result caused by measuring change on environment temperature, uncertainty in balance measurement results, uncertainty of measurement criteria used for detecting low background multi-channel $r$ energy spectrometer, uncertainty of the spectrometer system, uncertainty of standard soil sample activity for calibration and human factors.

Specific activities of radium-266, thorium-232 and potassium-40 measured with different detection times by using three different radioactive levels in the test room are shown in Table 1 to Table 3. Component analysis of uncertainty is carried out according to JJF1059.1-2012 Assessment of Uncertainty in Measurement and Representation ${ }^{[2]}$. The main factors that affect the uncertainty in the whole process are:

4.2.1.1 Components of relative standard uncertainty introduced by repeated detection of specific activity of radium-226, thorium-232 and potassium- 40 are expressed by $\mathrm{u}_{\mathrm{rRa}}$, $\mathrm{u}_{\mathrm{rTh}}$ and $\mathrm{u}_{\mathrm{rK}}$;

Relative standard uncertainty of sample repeat detection:

$$
u_{r}=\sqrt{\frac{1}{n(n-1)} \sum_{i=1}^{20}\left(d_{i}-\bar{d}\right)^{2}}
$$

Relative standard uncertainty of sample detection radium-226 $u_{r R a}=u_{R a} \bar{d}_{R a}$

Relative standard uncertainty of sample detection thorium-232 $u_{r T h}=u_{T h} \bar{d}_{T h}$

Relative standard uncertainty of sample detection potassium-40 $u_{r K}=u_{K} / \bar{d}_{K}$

4.2.1.2 Relative standard uncertainty introduced by balance indication error u1rel. The uncertainty of introduction of balance can be traced back to the measurement certificate. Expansion uncertainty of balance provided in the certificate is $10 \mathrm{~g}$ and $\mathrm{k}=2$. The maximum weighing range is $2000 \mathrm{~g}$, namely the standard uncertainty is $10 / 2=5 \mathrm{~g}$ and $\mathrm{k}=1$. The relative standard uncertainty of the balance $\mathrm{u}_{1 \mathrm{rel}}=5 \mathrm{~g} / 2000 \mathrm{~g}=0.25 \%$;

4.2.1.3 Uncertainties introduced by standard radioactive sources of radium-266, thorium-232 and potassium-40 have been given in the calibration certificate, and they are $4.8 \%, 4.4 \%$ and $4.4 \%(\mathrm{~K}=2)$ respectively. Therefore, the relative standard uncertainty of standard soil samples is: $\mathrm{u}_{2 \mathrm{rRa}}=2.4 \%, \mathrm{k}$ $=1 ; \mathrm{u}_{2 \mathrm{rTh}}=2.2 \%, \mathrm{k}=1 ; \mathrm{u}_{2 \mathrm{rK}}=2.2 \%, \mathrm{k}=1$.

4.2.1.4 Uncertainty of standard used for verifying low background multi-channel $\mathrm{r}$ energy spectrometer of testing equipment $\mathrm{Z} 18090015 \mathrm{u}_{3 \mathrm{rel}}$ and system uncertainty $\mathrm{u}_{4 \mathrm{rel}}$ 。

The verification certificate of low background multi-channel $r$ energy spectrometer shows that the degree of certainty of measurement standard is $7.6 \%$ and $\mathrm{k}=3$. It can be seen that the relative standard uncertainty $\mathrm{u}_{3 \mathrm{rel}}=2.53 \%$ and $\mathrm{k}=1$.

It can be seen from the verification certificate that system uncertainty of low background multi-channel $\mathrm{r}$ energy spectrometer is $10 \%$ and $\mathrm{k}=2$. When $\mathrm{k}=1$, the relative standard uncertainty $\mathrm{u}_{4 \mathrm{rel}}=5.00 \%$;

4.2.1.5 Uncertainty introduced by manual calculation. During calculation of the spectral line analysis, there will be an uncertainty component introduced due to different artificial calculation results of peak area. This part of the uncertainty component has been reflected in the repeated measurement of uncertainty assessment, so it is not assessed and takes 0 . 


\subsubsection{Calculation of measurement uncertainty}

There is no correlation between the above uncertainty components according to Table 4 below. According to the formulas (1) and (2), and JJF1059.1-2012, the related combined standard uncertainty of the three samples is calculated as per the following formulas (3) and (4):

Table.4 Statistics of the uncertainty components

\begin{tabular}{llll}
\hline Category for source of uncertainty & $\begin{array}{l}\text { Type } \\
\text { uncertainty }\end{array}$ & $\begin{array}{c}\text { Component involved } \\
\text { internal exposure index }\end{array}$ & $\begin{array}{c}\text { Component involved } \\
\text { external exposure index }\end{array}$ \\
\hline Relative standard uncertainty $u_{2}$ introduced by & & &
\end{tabular}

Relative standard uncertainty $u_{r R a}$ introduced by repeated detection of sample radium- 226

Relative standard uncertainty $u_{r T h}$ introduced by repeated detection of sample thorium-232

Type A

Relative standard uncertainty $u_{r K}$ introduced by repeated detection of sample potassium- 40

Relative standard uncertainty $u_{1 \text { rel }}$ introduced by indication error of balance

Relative standard uncertainty $u_{2 r R a}$ introduced by standard radioactive source of radium-226

Relative standard uncertainty $u_{2 r T h}$ introduced by standard radioactive source of thorium-232

Relative standard uncertainty $u_{2 r K}$ introduced by

standard radioactive source of potassium- 40

Type A

Relative standard uncertainty $u_{3 r e l}$ introduced by measurement standard

Relative standard uncertainty $u_{4 r e l}$ introduced by system deviation of low background multi-channel $r$ energy spectroscopy

$$
U_{r I_{R a}}=\sqrt{u_{r R_{a}}{ }^{2}+\left(u_{1 r e l}\right)^{2}+\left(u_{2 r R a}\right)^{2}+\left(u_{3 r e l}\right)^{2}+\left(u_{4 r e l}\right)^{2}}(3) U_{r I_{r}}=\sqrt{u_{R a}^{2}+u_{T h}^{2}+u_{K}^{2}+\left(u_{r e}\right)^{2}+\left(u_{r R}\right)^{2}+\left(u_{r r} T\right)^{2}+\left(u_{r l k}\right)^{2}+\left(u_{r r e}\right)^{2}+\left(u_{r r e l}\right)^{2}}
$$

If $\mathrm{k}=1$ specified in GB6566-2010 Standard,

The relative expansion uncertainty of internal exposure index of sample detection $U_{I_{R a}}=$ $1 \times U_{r I_{R a} a}$, the expansion uncertainty is $\mathrm{U}_{1}=U_{I_{R a}} \times \bar{d}_{I_{R a} / 100}$;

The relative expansion uncertainty of external exposure index of sample detection $U_{I_{r}=1 \times} U_{r I_{r}}$, the expansion uncertainty is $U_{2}=U_{I_{r} \times} \bar{d}_{I_{r} / 100}$.

Uncertainty assessment results of radioactive measurement for stone samples of three different radioactive levels by using three different detection times were shown in Table 5, Fig. 1 and Fig. 2:

Table. 5 Different stone samples with different testing time radioactive measurement uncertainty results summary table

\begin{tabular}{|c|c|c|c|c|}
\hline \multirow[t]{2}{*}{ Detection time/s } & \multirow[t]{2}{*}{ Category of uncertainty } & \multicolumn{3}{|c|}{$\begin{array}{l}\text { Measurement uncertainty of internal and external exposure index of 1-3\# } \\
\text { samples } / \%\end{array}$} \\
\hline & & $1 \#^{1}$ & $2 \#^{2}$ & $3 \#^{3}$ \\
\hline \multirow{4}{*}{600} & $U_{\text {rIRa }}$ & 7.67 & 6.31 & 6.16 \\
\hline & $U_{r I r}$ & 8.93 & 7.29 & 7.59 \\
\hline & $U_{I R a}$ & 7.67 & 6.31 & 6.16 \\
\hline & $U_{I r}$ & 8.93 & 7.29 & 7.59 \\
\hline \multirow{4}{*}{1800} & $U_{\text {rIRa }}$ & 6.78 & 6.30 & 6.14 \\
\hline & $U_{r I r}$ & 7.77 & 7.29 & 7.17 \\
\hline & $U_{I R a}$ & 6.78 & 6.30 & 6.14 \\
\hline & $U_{I r}$ & 7.77 & 7.29 & 7.17 \\
\hline \multirow{4}{*}{3600} & $U_{\text {rIRa }}$ & 6.38 & 6.30 & 6.12 \\
\hline & $U_{r I r}$ & 7.40 & 7.38 & 7.12 \\
\hline & $U_{I R a}$ & 6.38 & 6.30 & 6.12 \\
\hline & $U_{I r}$ & 7.40 & 7.38 & 7.12 \\
\hline
\end{tabular}

Note: 1. Levels of internal and external exposure results of sample are 0.12 and 0.50 respectively; 2. Levels of internal and external exposure results of sample are 0.50 and 1.00 respectively; 3 . Levels of internal and external exposure results of sample are 1.17 and 2.15 respectively; 


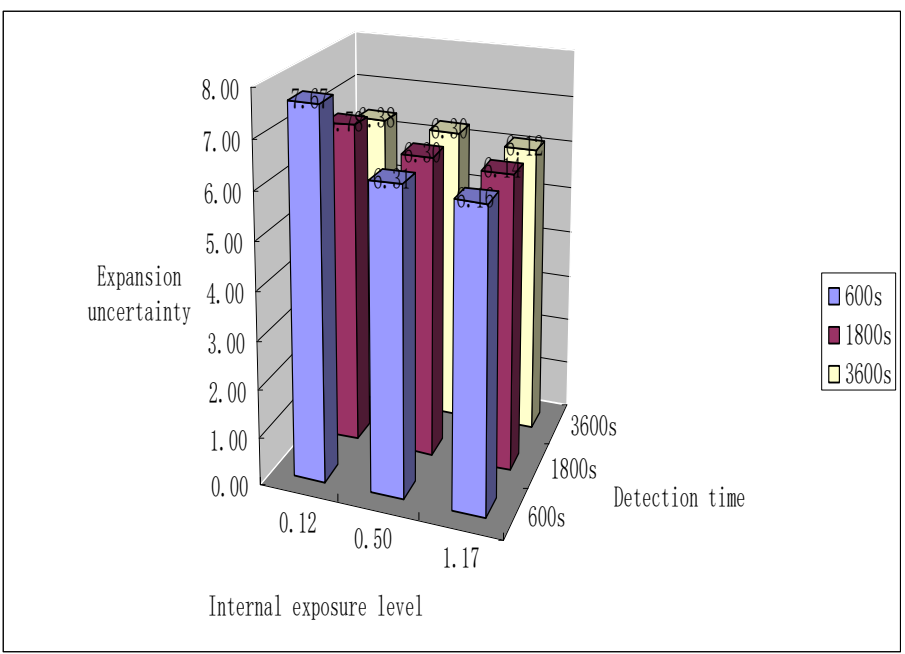

Fig.1 Relationship Among $\mathrm{UI}_{\mathrm{Ra}}, \mathrm{I}_{\mathrm{Ra}}$ and Detection Time $\mathrm{s}$

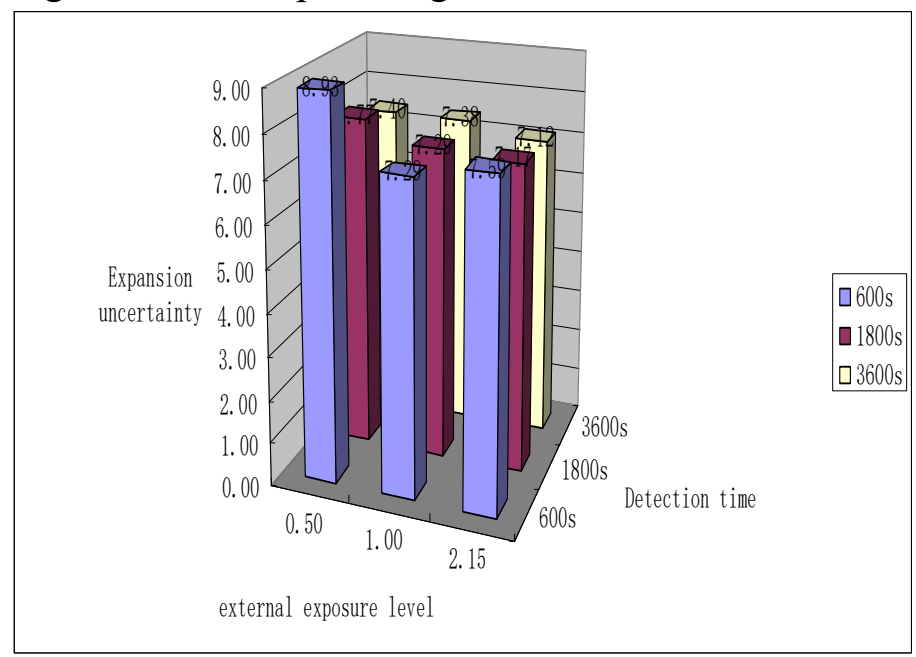

Fig.2 Relationship among $\mathrm{U}_{\mathrm{Ir}}, \mathrm{I}_{\mathrm{r}}$ and Detection Time $\mathrm{s}$

\section{Analysis and discussion}

1. Uncertainty introduced by repeated measurement, indication error of balance and other factors makes lower contribution to the uncertainty of measurement results from the calculation results, that is, it has little effect on dispersion of measurement results. Standard radioactive sources, standard equipment for calibration, system error of equipment and other factors with contribution on uncertainty of the results of more than $95 \%$ is the most important factor.

2. It can be obtained from Table 5, Fig. 1 and 2 that measurement uncertainties of internal and external exposure level obtained by using stone samples with three different radioactive levels (high, medium and low) and different detection times are less than that given in GB6566-2010 Standard $(\mathrm{K}=1,20 \%)$. Therefore, the measured data are reliable.

3. It can be obtained from Table 5, Fig. 1 and 2 that uncertainties of the 3 samples have increased slightly with reduction of internal and external exposure level of the samples themselves under the same detection time, but they are within the allowable range. Therefore, it can be considered that internal and external exposure level of the samples themselves has little impact on its measurement uncertainty within a reasonable range of internal and external exposure index, and the factor can not be considered.

4. In addition to $1 \#$ sample, measurement uncertainty the results of other two samples detected by using different detection times remains essentially unchanged, so it can be considered that the relationship between the dispersion time and the detection time is not significant when internal and external exposure level of the samples themselves is higher. However, if the internal and external exposure level is lower, the influence of detection time on dispersion of test results should be 
considered. It can be seen from test data in Table 1 that the ranges of internal and external exposure index for 20 times reach 0.10 and 0.25 respectively when 1 \# sample is detected for $600 \mathrm{~s}$, and the ratios between the ranges and the average values are $85 \%$ and $49 \%$. Therefore, detection time should not be too short in the actual test, especially when internal and external exposure level of the samples themselves is lower.

\section{Conclusion}

The above analysis shows that the main sources for uncertainty of the results include standard radioactive source, standard equipment for calibration and system error of equipment. And if the test time and other conditions are fixed, different internal and external exposure levels of the sample itself have little impact on dispersion and accuracy of its measurement result and influence of the factor can be ignored. It can be found from statistical data of the above charts that dispersion degree of results of stone detection data and measurement uncertainty can be stable when the detection time is at least 1800s or above. Therefore, it is recommended that the minimum test time should be no less than 1800 s when the radioactivity of natural stone samples is tested.

\section{References}

[1] Yang C, Zhang W, Liu X, et al. [The influence of the single different radiation dose and time on the microscopic structure and ultrastructure of Balb/c mice].[J]. Journal of Clinical Otorhinolaryngology Head \& Neck Surgery, 2014, 28(13):979-982.

[2] Fanucci E, Fiaschetti V, Rotili A, et al. Whole body 16-row multislice CT in emergency room: effects of different protocols on scanning time, image quality and radiation exposure[J]. Emergency Radiology, 2007, 13(5):251-257.

[3] Suzuki M, Nakano K, Suzuki K, et al. Influence of the sampling time on chromosomal aberrations at G2 phase in Syrian hamster embryonic cells irradiated with different types of radiation[J]. International Journal of Radiation Biology, 2000, 76(6):815.

[4] Stone R J, Michels J G, Wong S L, et al. Detection of terahertz radiation by hot electron effects in coupled quantum well photodiodes[J]. Applied Physics Letters, 1996, 69(23):3569-3571.

[5] Zhao F H, Dong H R, Zhou Y Y. The Detection and Analysis of Different Exterior Decorative Materials CS Panel Solar Radiation Performance Energy Saving Influence[J]. Applied Mechanics \& Materials, 2013, 368-370(1):1007-1012.

[6] Diehl J F. [Thiamine in irradiated foodstuffs I. Influence of different radiation conditions and of time after irradiation][J]. Zeitschrift für Le0bensmittel-Untersuchung und -Forschung, 1975, 157(6):317-321. 